\title{
A PSICOEDUCAÇÃO NA ADESÃO TERAPÊUTICA EM UTENTES COM ESQUIZOFRENIA: UMA SCOPING REVIEW
}

\author{
Tânia Vilar'; Maria José Nogueira²; Olga Valentim³; Paulo Seabra ${ }^{4}$ |
}

\section{RESUMO}

CONTEXTO: A esquizofrenia é uma doença crónica que afeta milhões de pessoas em todo o mundo. A não adesão aos projetos terapêuticos está presente em 50\% dos utentes com doença crónica.Torna-se necessário recorrer a uma intervenção que capacite os indivíduos a aderirem ao tratamento, sendo a intervenção mais indicada, a psicoeducação. A efetividade desta intervenção junto de pessoas com esquizofrenia é, no entanto, desconhecida com rigor.

OBJETIVO(S): Mapear os efeitos da psicoeducação na adesão terapêutica em pessoas com esquizofrenia.

MÉTODOS: Realização de uma scoping review, com base nas recomendações do Joanna Briggs Institute. Realizou-se uma pesquisa na plataforma EBSCO e nas bases de dados PubMed e Scielo em junho 2018, procurando-se estudos no período temporal de seis anos (2012 a 2018 ). RESULTADOS: Foram identificados 15 artigos e após a aplicação dos critérios de inclusão previamente definidos, foram selecionados três estudos para análise final. As intervenções realizadas nesses estudos foram: a aplicação do Nursing Psychoeducation Program; a visualização de filmes produzidos pela Alliance Psychoeducation Program e a realização de sessões de psicoeducação (com role - play). Em todas elas, usando medidas específicas antes e após as intervenções psicoeducacionais, verificou-se um aumento do conhecimento sobre a perturbação mental e uma mudança de atitude face à adesão ao regime medicamentoso.

CONCLUSÕES: Destaca-se a efetividade das técnicas psicoeducacionais em pessoas com esquizofrenia e salienta-se a relevância dos enfermeiros especialistas de saúde mental e psiquiátrica na equipa interdisciplinar no planeamento de intervenções psicoeducacionais.

PALAVRAS-CHAVE: Psicoeducação; Esquizofrenia; Enfermagem; Adesão terapêutica

\section{RESUMEN}

\section{“La psicoeducación en la adhesión terapéutica en usuarios con esquizofrenia: Una scoping review"}

CONTEXTO: La esquizofrenia es una enfermedad crónica que afecta a millones de personas en todo el mundo. La no adhesión a los proyectos terapéuticos está presente en el $50 \%$ de los pacientes con enfermedad crónica. Es necesario recurrir a una intervención que permita a las personas a adherirse al tratamiento, siendo la intervención más indicada, la psicoeducación. La efectividad de esta intervención en personas con esquizofrenia es, sin embargo, desconocida con rigor.

OBJETIVO(S): Mapear los efectos de la psicoeducación en la adhesión terapéutica en personas con esquizofrenia.

METODOLOGÍA: Realización de una scoping review, con base en las recomendaciones del Joanna Briggs Institute. Se realizó una investigación en la plataforma EBSCO y en las bases de datos PubMed y Scielo en junio de 2018, buscando estudios en el período temporal de seis años (2012 a 2018).

RESULTADOS: Se identificaron 15 artículos y después de la aplicación de los criterios de inclusión previamente definidos, se seleccionaron tres estudios para el análisis final. Las intervenciones realizadas en estos estudios fueron: la aplicación del Nursing Psychoeducation Program; la visualización de películas producidas por la Alliance Psychoeducation Program y la realización de sesiones de psicoeducación (con rol - play). En todas ellas, tras la aplicación de medidas específicas antes y después de las intervenciones psicoeducativas, se verificó un aumento del conocimiento sobre la perturbación mental y un cambio de actitud frente a la adhesión al régimen medicamentoso.

CONCLUSIONES: Se destaca la efectividad de las técnicas psicoeducativas en personas con esquizofrenia y se destaca la relevancia de los enfermeros especialistas de salud mental y psiquiátrica en el equipo interdisciplinario en la planificación de intervenciones psicoeducativas.

\section{DESCRIPTORES: Psicoeducación; Esquizofrenia; Enfermería; Adhesión terapéutica}

\section{ABSTRACT \\ "The effects of psychoeducation on therapeutic adherence in people with schizophrenia: A scoping review"}

BACKGROUND: Schizophrenia is a chronic that affects millions of people around the world. Non-adherence to therapeutic projects is present in 50\% of patients with chronic disease. It is necessary to resort to an intervention that enables individuals to adhere to treatment, being the most indicated intervention, psychoeducation. The effectiveness of this intervention among people with schizophrenia is, however, strangely unknown.

AIM: To map the effects of psychoeducation on therapeutic adherence in people with schizophrenia.

METHODS: Scoping review, based on the recommendations of Joanna Briggs Institute. A survey was conducted on the EBSCO platform and the PubMed and Scielo databases in June 2018, looking for studies over the six-year timeframe (2012 to 2018).

RESULTS: Fifteen articles were identified and after the application of the previously defined inclusion criteria, three studies were selected for final analysis. The interventions carried out in these studies were: the application of the Nursing Psychoeducation Program; viewing films produced by the Alliance Psychoeducation Program and conducting psychoeducation sessions (with role - play). Using specific measures before and after psychoeducational interventions, for all studies, there was an increase knowledge about mental disorder, as well, a change in patient's compliance.

CONCLUSIONS: The effectiveness of psychoeducational techniques in people with schizophrenia is discussed, as well the relevance of the mental and psychiatric nurse's psychoeducational interventions in the scope of the health care team.

\section{KEYWORDS: Psychoeducation; Schizophrenia; Nursing; Pa- tient compliance}

Submetido em 30-12-2018

Aceite em 01-04-2019

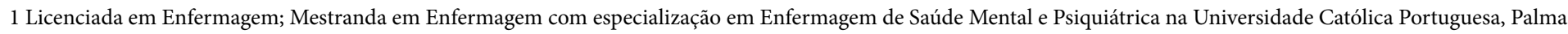
de Cima, Lisboa, Portugal, vilar1992@hotmail.com

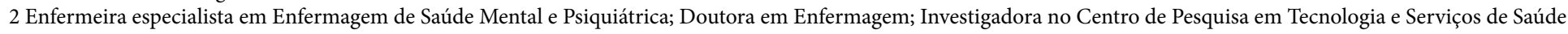
(CINTESIS - NursID); Professora Adjunta na Escola Superior de Saúde Atlântica, Barcarena, Portugal, nogueira.mjc@gmail.com

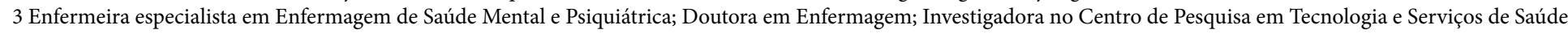
(CINTESIS - NursID); Professora Adjunta na Escola Superior de Saúde Atlântica, Barcarena, Portugal, ommvalentim2@gmail.com

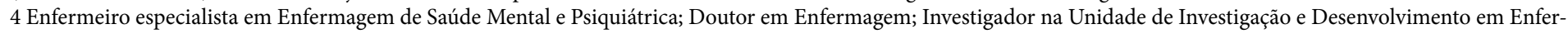
magem (UI\&DE); Professor Adjunto na Escola Superior de Enfermagem, Lisboa, Portugal, pauloseabra@esel.pt

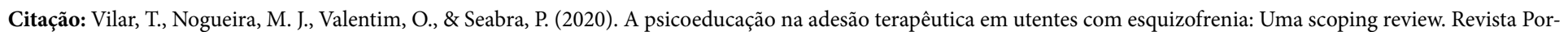
tuguesa de Enfermagem de Saúde Mental (Spe7), 103-108. 


\section{INTRODUÇÃO}

A esquizofrenia é uma doença crónica que afeta quase 24 milhões de pessoas em todo o mundo (World Health Organization [WHO], 2012, citada por Chagani, 2015).

Esta patologia abrange um conjunto de variadas características que provocam alterações na pessoa a diversos níveis, a associar ainda ao estigma social existente (Galuppi, Turola, \& Mazzoni, 2012, citados por Ngoc, Weiss, \& Trung, 2016).

Muitos utentes com esquizofrenia necessitam de estabilização duradoura com medicação para prevenir recaídas, contudo, a não - adesão está presente em $50 \%$ dos utentes com doenças crónicas (WHO, 2012, citada por Al Batran, 2015), independentemente da sua etiologia.

Entende-se por adesão o comportamento de uma pessoa coincidente com as recomendações de um prestador de cuidados de saúde (Ordem dos Enfermeiros, 2009). Isto é, cumpre indicações relativas à toma da medicação, no cumprimento dos ensinos/consultas, na procura de cuidados de saúde e nas mudanças dos estilos de vida.

O comprometimento da adesão é influenciado pela relação entre o utente e o profissional (Day et al., 2005, citados em Matsuda \& Kohno, 2016); pelos efeitos secundários dos anti-psicóticos (Dibonaventura, Gabriel, Dupclay, Gupta \& Kim, 2012; Eticha, Teklu, Ali, Solomon \& Alemayehu, 2015, citados por Matsuda \& Kohno, 2016); pelas crenças acerca dos efeitos da medicação (Higashi et al., 2013, citado por Matsuda \& Kohno, 2016); pelo reduzido insight e conhecimento acerca dos sinais e sintomas da sua patologia. (Barkhof, Meijer, Sonneville, Linszen, \& Haan, 2012; Higashi et al., 2013; Novick et al., 2015, citados por Matsuda \& Kohno, 2016).

As estimativas do incumprimento das prescrições farmacológicas e não farmacológicas são significativas, revelando-se um desafio diário, para os profissionais de saúde (Byrne, Cardoso, \& Xavier, 2016).

Assim, torna-se essencial intervir de forma a capacitar o indivíduo para aderir ao tratamento. A medicação, as sessões de aconselhamento e educação, podem ser implementadas, contudo, de todas as intervenções, a mais promissora é a Psicoeducação (Bauml, Frobose, Kraemer, Rentrop, \& Pitschel-Walz, 2006, citados por Matsuda \& Kohno, 2016). O termo 'Psicoeducação' surgiu na área da Psiquiatria (com George Brown, 1972), através de trabalhos desenvolvidos com familiares de utentes com esquizofrenia e mais tarde foi desenvolvida com Carol Anderson em 1980, como tratamento adjuvante da esquizofrenia (Srivastava \& Panday, 2016). A Psicoeducação é uma intervenção psicoterapêutica designada para promover o coping nos utentes e familiares (Lincoln, Wilhelm, \& Nestoriuc, 2007, citado em Srivastava \& Panday, 2016) e é considerada um processo que permite ensinar os utentes com perturbação mental e as suas famílias, sobre a natureza da doença (etiologia, progressão, consequências, prognóstico e alternativas de tratamento) (Barker, 2003, citado em Srivastava \& Panday, 2016). No que se refere aos benefícios da Psicoeducação junto de pessoas com esquizofrenia, verifica-se que é desconhecida com rigor, o que torna relevante a realização de uma revisão de literatura com o objetivo de sintetizar e sistematizar o conhecimento existente acerca dos efeitos da psicoeducação em pessoas com esquizofrenia.

Face a esta evidência, surge a questão: Quais os efeitos da psicoeducação na adesão terapêutica em pessoas com esquizofrenia?

\section{MÉTODOS}

A metodologia usada para esta revisão tem por base o Manual de Joanna Briggs Institute (Peters et al, 2015), considerando os passos para a realização de uma scoping review: (1) Identificar a questão de pesquisa; (2) Identificar os estudos relevantes; (3) Selecionar os estudos; (4) Mapear os dados; (5) Recolher, sintetizar e relatar os resultados.

Primeiramente, foi definida a questão de pesquisa (Passo 1), baseada no PCC (População, Conceito e Contexto), assim como os critérios de inclusão:

P - População: pessoas adultas, com idades compreendidas entre os 18 e os 65 anos, portadoras de esquizofrenia;

C - Conceito: psicoeducação, enquanto intervenção psicoterapêutica;

C - Contexto: qualquer contexto.

Para esta revisão foram considerados estudos primários, revisões de literatura e literatura cinzenta. Posteriormente, de forma a identificar-se os estudos relevantes (Passo 2), que pudessem dar resposta à questão de investigação, foi realizada uma pesquisa desde $1 \mathrm{a}$ 16 de junho de 2018. Numa primeira fase, foi realizada uma pesquisa na plataforma EBSCO, de forma a encontrar-se os termos de pesquisa mais usados nos artigos, que melhor servissem o propósito da pesquisa. 
Os termos de pesquisa selecionados foram: psychoeducation e intervention e os descritores (DeCS e MeSH) nursing, schizophrenia e patient compliance.

Numa segunda fase, como estratégia de pesquisa, foi realizada uma pesquisa em duas bases de dados distintas: Pub Med (usados os descritores MeSH) e SciELO e na plataforma EBSCO (usados os descritores DeCS), com os seguintes termos e operadores booleanos: 'Psychoeducation' AND 'Patient compliance OR adherence' AND 'schizophrenia' AND 'intervention OR treatment OR therapy' AND 'nursing OR nurses OR nurse OR nursing care OR nursing practice'. Para os termos psychoeducation e schizophrenia, a pesquisa foi delimitada por: Título; período de seis anos (2012 2018); artigos na língua portuguesa, inglesa e espanhola; e artigos disponíveis em texto integral de livre acesso.

Os estudos foram selecionados (Passo 3) e os dados foram mapeados (Passo 4), analisando os títulos e os resumos, baseado nos critérios de inclusão previamente definidos. Da pesquisa efetuada obteve-se um total de 15 artigos: 2 deles foram excluídos por serem duplicados; 6 foram excluídos pelo título quer por o grupo alvo não corresponder aos critérios de inclusão, quer por não focarem a psicoeducação, enquanto intervenção psicoterapêutica, para a adesão terapêutica; 4 artigos foram excluídos pelo resumo, quer por serem tipos de revisões de literatura que não se enquadravam nos critérios de inclusão, quer por não incidirem em específico na psicoeducação. Assim sendo, foram incluídos 3 artigos para a análise integral, os quais cumpriam os critérios de inclusão.

\section{RESULTADOS}

De modo a atingir-se o Passo 5 recomendado pelo Manual de Joanna Briggs Institute (Peters et al, 2015), é apresentada de seguida, no Quadro 1, a síntese dos dados recolhidos nos artigos incluídos na revisão.

Quadro 1 - Síntese dos artigos incluídos na revisão

\begin{tabular}{|c|c|c|c|c|}
\hline $\begin{array}{l}\text { Autor (es) } \\
\text { Ano, Nome do artigo }\end{array}$ & $\begin{array}{l}\text { Desenho de Estudo } \\
\text { e Amostra }\end{array}$ & Objetivo do estudo & $\begin{array}{l}\text { Intervenções Psicoedu- } \\
\text { cacionais }\end{array}$ & Resultados \\
\hline $\begin{array}{l}\text { Matsuda e Kohno (2016) } \\
\text { Effects of the Nurs- } \\
\text { ing Psychoeducation } \\
\text { Program on the Ac- } \\
\text { ceptance of Medication } \\
\text { and Condition - Specific } \\
\text { Knowledge of Patients } \\
\text { with Schizophrenia }\end{array}$ & $\begin{array}{l}\text { Quase experimental } \\
43 \text { utentes (idade } \\
\geq 20 \text { ) com esquizo- } \\
\text { frenia } \\
\text { (dois hospitais } \\
\text { psiquiátricos do } \\
\text { Japão) }\end{array}$ & $\begin{array}{l}\text { Avaliar a utilidade clínica de um } \\
\text { programa de psicoeducação (NPE } \\
\text { - Nursing Psychoeducation Pro- } \\
\text { gram) na adesão à medicação e no } \\
\text { aumento do conhecimento sobre a } \\
\text { patologia e efeitos secundários da } \\
\text { medicação. }\end{array}$ & $\begin{array}{l}\text { * Aplicação do pro- } \\
\text { grama NPE (dirigido } \\
\text { por } 2 \text { enfermeiros com } \\
\text { formação no programa); } \\
\text { * Aplicação de instru- } \\
\text { mentos de avaliação an- } \\
\text { tes e após a intervenção } \\
\text { para avaliar a evolução. }\end{array}$ & $\begin{array}{l}\text { * Maior score nos questionários } \\
\text { (sobre medicação) aplicados após a } \\
\text { intervenção de psicoeducação; } \\
\text { ^ O NPE é útil para promover a } \\
\text { adesão à medicação em utentes com } \\
\text { esquizofrenia, mas não contribui dire- } \\
\text { tamente para o aumento do conheci- } \\
\text { mento da patologia. }\end{array}$ \\
\hline $\begin{array}{l}\text { Maffei, Gorges, Kissling, } \\
\text { Schreiber, e Rummel- } \\
\text { Kluge } \\
\text { (2015) } \\
\text { Using films as a psycho- } \\
\text { education tool for pa- } \\
\text { tients with schizophre- } \\
\text { nia: a pilot study using a } \\
\text { quasi - experimental pre } \\
\text { - post design }\end{array}$ & $\begin{array}{l}\text { Quase experimental } \\
102 \text { utentes } \\
\text { (média de idades } \\
\text { de } 35,1 \text { anos) com } \\
\text { esquizofrenia (um } \\
\text { hospital psiquiátri- } \\
\text { co na Alemanha) }\end{array}$ & $\begin{array}{l}\text { Testar a viabilidade e uma nova } \\
\text { forma de psicoeducação (uso de } \\
\text { filmes) em utentes com esquizo- } \\
\text { frenia. }\end{array}$ & $\begin{array}{l}\text { * Visualização de } 6 \\
\text { filmes, produzidos pela } \\
\text { Alliance Psychoeduca- } \\
\text { tion Program (presença } \\
\text { sempre de um enfer- } \\
\text { meiro): } \\
\text { - Sessão de } 35 \text { minutos } \\
\text { (17 minutos de filme + } \\
18 \text { minutos de debate). } \\
\text { * Aplicação de escalas } \\
\text { antes e após a inter- } \\
\text { venção para avaliar a } \\
\text { evolução }\end{array}$ & $\begin{array}{l}\text { * Maior score nas escalas de avaliação } \\
\text { após a intervenção da psicoeducação: } \\
\text { - Aumento do conhecimento acerca } \\
\text { da medicação e da patologia; } \\
\text { - Aumento do insight para a doença; } \\
\text { - Mudança de perceção para a quali- } \\
\text { dade de vida. } \\
\text { * A perceção dos efeitos secundários } \\
\text { da medicação não diminuiu. } \\
\text { * A frequência e a duração das (re) } \\
\text { admissões no hospital, não mudaram } \\
\text { significativamente após a intervenção. } \\
\text { * Os utentes, após alguns meses da } \\
\text { aplicação da intervenção, ainda se } \\
\text { recordavam das informações que } \\
\text { receberam. }\end{array}$ \\
\hline $\begin{array}{l}\text { Choe, Sung, Kang, e Yoo } \\
\text { (2015) } \\
\text { Impact of Psychoeduca- } \\
\text { tion on Knowledge of } \\
\text { and Attitude Toward } \\
\text { Medications in Clients } \\
\text { With Schizophrenia and } \\
\text { Schizoaffective Disorders }\end{array}$ & $\begin{array}{l}\text { Quase experimental } \\
61 \text { utentes } \\
\text { ( } 35 \text { com esquizo- } \\
\text { frenia e } 26 \text { com } \\
\text { perturbação esqui- } \\
\text { zoafetiva, de um } \\
\text { hospital psiquiátri- } \\
\text { co da Coreia do Sul) }\end{array}$ & $\begin{array}{l}\text { Testar a viabilidade e uma nova } \\
\text { forma de psicoeducação (uso de } \\
\text { filmes) em utentes com esquizo- } \\
\text { frenia. } \\
\text { Avaliar os efeitos da intervenção } \\
\text { psicoeducacional focada na pro- } \\
\text { moção de conhecimento e atitudes } \\
\text { face à medicação anti - psicótica } \\
\text { em utentes com esquizofrenia (ou } \\
\text { com perturbação esquizoafetiva). }\end{array}$ & $\begin{array}{l}\text { * Sessão de Psicoedu- } \\
\text { cação (conduzida por } 2 \\
\text { enfermeiras com forma- } \\
\text { ção própria na área): } \\
\text { - } 6 \text { semanas de psico- } \\
\text { educação ( } 1 \text { sessão por } \\
\text { semana) } \\
\text { - Uso de material áudio } \\
\text { - visual para comple- } \\
\text { mentar a sessão. }\end{array}$ & $\begin{array}{l}\text { * Maior score nas escalas de avaliação } \\
\text { após a intervenção da psicoeducação: } \\
\text { - Aumento significativo do conheci- } \\
\text { mento sobre a patologia; } \\
\text { - Melhoria significativa da atitude face } \\
\text { à medicação. }\end{array}$ \\
\hline
\end{tabular}




\section{DISCUSSÃO}

A análise dos artigos selecionados para a revisão, permitiu examinar diferentes formas de aplicação da psicoeducação. Apesar das três abordagens serem distintas, todas elas apresentavam a mesma base de aplicação, focando-se nos principais temas a abordar nas sessões psicoeducacionais: conhecimento da esquizofrenia (definição, causas, sintomas); conhecimento da medicação (mecanismo de ação e efeitos secundários) e estratégias de coping para lidar com a patologia.

Os três estudos evidenciaram a importância da presença do enfermeiro com formação específica na área de saúde mental e psiquiatria, conduzindo as sessões, e apontando a relação enfermeiro - utente como sendo um dos fatores influenciadores do sucesso da sessão. Como nos mostra a evidência: a não adesão é influenciada pela relação entre o utente e o profissional (Day et al, 2005, citados por Matsuda \& Kohno, 2016).

No estudo de Matsuda e Kohno (2016), em que se aplicou um programa psicoeducacional de enfermagem (NPE - Nursing Psychoeducation Program), ainda que houvesse um aumento de conhecimentos e uma mudança de atitude face à medicação, o programa não contribuiu diretamente para aumentar o conhecimento sobre a esquizofrenia, facto referido no estudo pela possibilidade de ser influenciado pelo insight dos indivíduos face à sua situação e pelo prévio conhecimento do tema.

Ainda assim, tem-se tornado cada vez mais evidente que a aquisição de conhecimentos é necessária, mas não o suficiente para a modificação comportamental em utentes com esquizofrenia. Mudar as atitudes dos utentes é mais importante que promover o seu conhecimento (Ministry of Health, Labour and Welfare, 2000, citados por Matsuda \& Kohno, 2016).

Com os resultados deste estudo, através da aplicação de instrumentos de avaliação (MPS - Medication Perception Scale for Patients with Schizophrenia; DAI - Drug Attitude Inventory - 10 Questionnaire; KIDI - Knowledge of Psychiatric Illness And Drugs Inventory), tornou-se claro que, em utentes em contexto de internamento (o próprio estudo refere como limitação não abranger a área do ambulatório), a intervenção psicoeducacional tem repercursões significativas na atitude face à adesão à medicação.

A acrescentar ainda que o papel do enfermeiro foi evidenciado, na medida em que o programa foi conduzido por dois especialistas na área de saúde mental e psiquiatria, tendo tido formação específica no programa NPE (com acesso a leituras, exemplos práticos de psicoeducação através de meios audiovisuais e 'role - play').

Futuramente torna-se necessário determinar o efeito a longo prazo do programa psicoeducacional, visto o NPE ter sido aplicado durante 4 dias.

No estudo de Maffei, Gorges, Kissling, Schreiber, \& Rummel-Kluge (2015), em que a intervenção psicoeducacional teve por base a visualização de 6 filmes (3 filmes por semana, durante 2 semanas), também os resultados foram significativos. Neste estudo, os resultados mostraram que houve um aumento do conhecimento acerca da medicação e da própria patologia, do insight para a doença e da perceção para a qualidade de vida (mas não da perceção negativa sobre os efeitos secundários da medicação), contrariamente ao estudo de Matsuda e Kohno (2016) que mostrou resultados ao nível da adesão à medicação e não tão significativos no aumento do conhecimento sobre a esquizofrenia. Tal como no estudo de Matsuda e Kohno (2016), também este recorreu ao uso de instrumentos de avaliação para avaliar os momentos antes e após a intervenção psicoeducacional: MARS - Medication Adherence Rating Scale; The Knowledge Of Illness about Schizophrenia Questionnaire e a Visual Analogue Scale (para a perceção de qualidade de vida).

Além disso, dois meses após a alta dos utentes, verificou-se que ainda mantinham os conhecimentos bem assentes. Os dois meses iniciais após a alta clínica são fulcrais para a adesão ao tratamento e redução de readmissões (Markowitz, Karve, Panish, Candrilli,\& Alphs, 2013, citados por Maffei et al., 2015).

Assim, contrariamente aos estudos de Matsuda e Kohno (2016) e de Choe et al (2015), o estudo de Maffei et al (2015) investiu não só no acompanhamento do utente no momento do internamento, como no pós alta, o que foi um fator importante na medida em que, há resultados que evidenciam a influência da psicoeducação na taxa de recaídas e readmissões (Pekkala \& Merinder, 2002, citado por Choe et al., 2015).

O estudo de Choe et al (2015) também teve uma abordagem psicoeducacional que trouxe repercurssões no utente com esquizofrenia, semelhantes aos estudos anteriores, nomeadamente no aumento significativo do conhecimento sobre a patologia e na mudança de atitude face à medicação, tendo este último estudo, o maior período temporal de aplicação da intervenção psicoeducacional - 6 semanas. Mais uma vez existiu a aplicação de instrumentos de avaliação antes e após a sessão de psicoeducação: DAI - Drug Attitude Inventory - 10 Questionnaire e o Knowledge Instrument. 
Contudo, tal como nos outros estudos, também este envolveu uma amostra não representativa da população (limitação referida). Ainda assim, os três estudos traduzem a importância da Psicoeducação para a adesão terapêutica nos utentes com esquizofrenia, sendo que o estudo de Maffei et al (2015) faz a anotação de abranger também a família. A importância da educação aos familiares tem sido demonstrada em diversos estudos, todavia ainda é uma área em crescimento, pelo estigma e exclusão social existente na área da saúde mental (Bauml et al., 2006; McFarlane, Dixon, Lukens, \& Lucksted, 2003; Farrely et al., 2014, citados por Maffei et al., 2015).

\section{CONCLUSÕES}

Partindo da questão de investigação quais os efeitos da psicoeducação na adesão terapêutica em pessoas com esquizofrenia? os estudos demonstram que a intervenção Psicoeducacional permite o aumento de conhecimentos, no utente com esquizofrenia, sobre a sua doença e a necessidade de aderir ao regime medicamentoso.

A Psicoeducação permite a mudança de comportamentos face à adesão à medicação e à perceção sobre a doença mental.

Além disso, paralelamente à intervenção psicoeducacional, durante o período de internamento, é igualmente necessário um seguimento na pós-alta e o envolvimento da família do utente com esquizofrenia, de forma a aumentar a adesão por parte do utente e, consequentemente, reduzir as taxas de recaída e readmissões.

Destaca-se a relevância dos enfermeiros especialistas de saúde mental e psiquiátrica na equipa interdisciplinar no planeamento de intervenções psicoeducacionais.

\section{IMPLICAÇÕES PARA A PRÁTICA CLÍNICA}

Futuramente, torna-se essencial realizar estudos que abarquem uma amostra representativa do universo de utentes, de tal forma que permitam a generalização dos resultados para a população.

Torna-se também necessário investir nos estudos sobre a psicoeducação na área concreta da esquizofrenia e do ambulatório, apostando-se na adesão ao tratamento dos utentes com esta doença crónica (com envolvimento também da família) e apostando-se no futuro da profissão e nos cuidados de enfermagem de saúde mental e psiquiatria na comunidade
É necessário continuar a investir na pesquisa neste campo de atuação, considerando a necessidade de estudos em Portugal.

\section{REFERÊNCIAS BIBLIOGRÁFICAS}

Al Batran, M. (2015). Evidence Based Practice: The Effectiveness of Group Psychoeducation for medications adherence among inpatient adults with schizophrenia in psychiatric and mental health settings. Middle East Journal of Nursing, 9(2), 25-30. Dispnível em: https:// pdfs.semanticscholar.org/b43d/fbc8a81343091d3dd2b7911ddab98cd0a515.pdf

Byrne, M., Cardoso, A., \& Xavier, M. (2016). Adesão ao tratamento nas perturbações psiquiátricas: $\mathrm{o}$ impacto das atitudes e das crenças em profissionais de serviços de psiquiatria e saúde mental em Portugal. Parte I: aspetos conceptuais e metodológicos. Revista Portuguesa de Saúde Pública, 34, 209-219. Doi: 10.1016/j.rpsp.2016.05.004

Chagani, S. (2015). Psychoeducation - A Mental Health Promoting Practice in Schizophrenia. Journal of Pioneering Medical Sciences, 5(1), 3-6. Disponível em: https://pdfs.semanticscholar.org/ e652/3a431711a69842ccbc910c1e8315c6d72526. pdf?_ga $=2.188182560 .965037712 .1572908589$ 1846501729.1572908589

Choe, K., Sung, B.J., Kang Y., \& Yoo S.Y. (2016). Impact of Psychoeducation on Knowledge of and Attitude Toward Medications in Clients With Schizophrenia and Schizoaffective Disorders. Perspectives in Psychiatric Care, 52(2), 113-119. Doi:10.1111/ppc.12106

Maffei, C., Gorges, F., Kissling, W., Schreiber, W., \& Rummel-Kluge, C. (2015). Using a film as a psychoeducation tool for patients with schizophrenia: a pilot study using a quasi-experimental pre-post design. BMC Psychiatry, 15, 1-7. Doi: 10.1186/s12888-0150481-2

Matsuda, M. \& Kohno, A. (2016). Effects of the Nursing Psychoeducation Program on the Acceptance of Medication and Condition - Specific Knowledge of Patients with Schizophrenia. Archives of Psychiatric Nursing, 30(5), 581-586. Doi: 10.1016/j.apnu.2016.03.008 
Ngoc T.N., Weiss B., \& Trung, L.T. (2016). Effects of the Family Schizophrenia Psychoeducation Program for Individuals with Recent Onset Schizophrenia in Viet Nam. Asian Journal of Psychiatry, 22, 162-166. Doi:10.1016/j.ajp.2016.06.001

Ordem dos Enfermeiros. (2009). Estabelecer Parcerias com os Indivíduos e as Famílias para Promover a Adesão ao Tratamento. Lisboa: Autor.

Peters, M., Godfrey, C., McInerney, P., Soares, C. B., Khalil, H., \& Parker, D. (2015). Methodology for jbi scoping reviews. In E. Aromataris (Ed.), The Joanna Briggs Institute Reviewers manual 2015 (3 - 24). South Australia: The Joanna Briggs Institute.
Srivastava P. \& Panday R. (2016). Psychoeducation an Effective Tool as Treatment Modality in Mental Health. International Journal of Indian Psychology, 4(82), 123-130. Disponível em: https://ijip.in/wp-content/uploads/ArticlesPDF/article_d03dca6899bbb3be3b0014ee8e7f1011.pdf 